\title{
The Inter-Relation between Solitary Kidney and Diabetes Mellitus-What Patterns are Known?
}

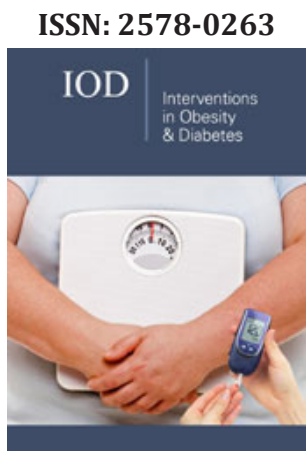

*Corresponding author: Oana Milas, Assistant Professor, Department of Nephrology, Victor Babeș University of Medicine and Pharmacy, Timișoara, Romania

Submission: 眥 December 23, 2020

Published: 笽January 21, 2021

Volume 4 - Issue 5

How to cite this article: Florica Gadalean, Oana Milas, Adalbert Schiller, Cristina Gluhovschi, Adelina Mihaescu, et al. The Inter-Relation between Solitary Kidney and Diabetes Mellitus-What Patterns are Known?. Interventions Obes Diabetes 4(5). IOD.000597. 2021.

DOI: 10.31031/IOD.2021.04.000597

Copyright@ Oana Milas. This article is distributed under the terms of the Creative Commons Attribution 4.0 International License, which permits unrestricted use and redistribution provided that the original author and source are credited.

\author{
Florica Gadalean ${ }^{1,2,3,4}$, Oana Milas ${ }^{1,2,3,4 *}$, Adalbert Schiller ${ }^{1,2,3,4}$, Cristina \\ Gluhovschi1,2,3,4, Adelina Mihaescu1 ${ }^{1,2,3,4}$, Flaviu Bob ${ }^{1,2,3,4}$ and Ligia Petrica ${ }^{1,2,3,4,5,6}$ \\ ${ }^{1}$ Dept. of Nephrology, \\ ${ }^{2}$ Victor Babes' University of Medicine and Pharmacy Timisoara, Romania \\ ${ }^{3}$ County Emergency Hospital Timisoara, Romania \\ ${ }^{4}$ Centre for Molecular Research in Nephrology and Vascular Disease, Romania \\ ${ }^{5}$ Center for translational Research and Systems Medicine, Romania \\ ${ }^{6}$ Center for Cognitive Research in Neurologic and Psychiatric Disease,Romania
}

Abstract

Over the last 20 years, most of the studies regarding the kidney outcome of people with solitary kidney (SK) show that there is a progressive loss of renal function in these individuals. In order to carry out the renal work undertaken by two kidneys, SK must adapt through complex phenomena of hypertrophy and hyperfiltration. The adaptive changes are considered, "the first hit" of SK because these lead to glomerular injury with progressive loss of kidney function. Thus, more than $50 \%$ of children with congenital SK have markers of renal injury by the age of 18 years. Also, living kidney donors have 3 to 5 times higher relative risk of end stage renal disease (ESRD as compared with their counterparts with two kidneys. A "second hit", such as obesity, hypertension, and diabetes mellitus (DM), which superimpose to the SK status conducts to a faster decline in kidney function and to a poor renal prognosis of these individuals. Due to the fact that type 2 DM is a pandemic disease of the 21st century, with an increasing prevalence among SK patients, in this paper we aimed to present different patterns of the inter-relation between SK and DM.

Keywords: Acquired solitary kidney; Congenital solitary kidney;Diabetes mellitus;Living kidney donors

Abbreviations: ASK: Acquired Solitary Kidney; CKD: Chronic Kidney Disease; CSK: Congenital Solitary Kidney; DM: Diabetes Mellitus; ESRD: End Stage Renal Disease; LKD: Living Kidney Donor; SK: Solitary Kidney; UNX: Uninephrectomy

\section{Introduction}

Data from experimental studies shows that diabetes mellitus (DM) and uninephrectomy (UNX) in animals with induced DM had a cumulative effect on renal hypertrophy, with increased value of the glomerular sclerosis index [1-4]. Moreover, it seems that the acceleration of glomerular lesions was more closely related to hemodynamic adaptations than to glomerular hypertrophy [1-3]. The negative consequences which result from the inter-relation of DM with UNX revealed in experimental studies were also described in human studies, mainly in people who donated one kidney.

\section{Living Kidney Donors and Diabetes Mellitus}

Acquired solitary kidney (aSK) in adults is mainly due to radical UNX for living kidney donation, renal tumor, and trauma. To date, globally, 35,000 living kidney donor (LKD) transplants are performed yearly [5]. Statistical data for the United States shows that the rate of living kidney donation had progressively increased from 1,800 donations in 1998 to 6,600 donations in 2004. Since 2011, the annual rate of living kidney donation has decreased and has remained stable around 5,650 kidney donations per year [6]. Although in the past UNX for living kidney donation was regarded as a safe condition, nowadays it is well-established that donors have a higher risk of end stage renal disease (ESRD) than matched healthy non-donors [7]. Thus, following UNX, LKD have 3 to 5 times higher relative risk of ESRD as compared with their counterparts with two kidneys $[8,9]$. It has been shown that DM and hypertension are the most frequent causes of ESRD at over 10 years after donation $[8,9]$. 
In the general population, DM accounts for nearly half of all ESRD cases, while in living kidney donors (LKD), $12 \%$ to $22 \%$ of ESRD cases have been related to DM $[9,10]$. This lower percentage of ESRD in relation to DM in LKD seems to be due to the fact that those individuals who are at very high risk of DM are excluded from donation. Moreover, LKD are considered to be healthier $[6,9,11]$. Diabetes mellitus develops in 3-18\% of LKD at 5-18 years after donation and is strongly associated with an increase in the incidence of ESRD from the early to the late post-donation period [11]. In a large cohort which consisted of 125,427 LKD, the cumulative incidence of ESRD increased from 10 cases with ESRD per 10,000 at 10 years post donation to 85 cases with ESRD cases per 10,000 at 25 years post-donation, late post-donation ESRD being more frequently reported as diabetic-ESRD [8]. When LKD who develop DM were compared with diabetic patients with two kidneys, the first ones were more likely to superimpose hypertension and proteinuria [11]. In LKD who associate DM, it seems that eGFR decline exceeds that of non-diabetic counterparts only if they have hypertension and proteinuria, an observation which is similar to what is known from the general population [11]. By contrast, other studies revealed that the prevalence of proteinuria and hypertension in diabetic LKD is not different from what is found in diabetic patients with two kidneys [12]. It is conceivable that since compensatory hyperfiltration is almost complete in the early postdonation period, no boost hyperfiltration exists by the time when DM occurs. Moreover, adaptive hyperfiltration in the remaining kidney is maintained stable through compensatory glomerular hypertrophy, with augmentation in renal plasma flow and in the whole-kidney ultrafiltration coefficient, rather than through an increase in the intraglomerular pressure, as described in DM [13].

\section{Surgically Acquired Solitary Kidney (SaSK) and Diabetes Mellitus}

In a recent retrospective study performed on 172 patients with SaSK, DM was an independent risk factor for chronic kidney disease (CKD) stage 3, with a nearly four-fold higher risk (OR 4.401, 95\% CI 1.693-11.44, p=0.002) [14]. Similar findings were described in a 6.3 year follow-up study conducted on a cohort of 499 SaSK in which, after nephrectomy, DM was associated with low-eGFR (OR=8.96, $\mathrm{P}=0.002)$ and with high serum creatinine $(\mathrm{OR}=10, \mathrm{P}<0.0001)$ [15]. In a small clinical series, Nielsen et al observed that patients with SaSK and DM are at an increased risk of developing diabetic nephropathy with nephrotic range proteinuria [16]. Similar findings were reported by Samspon et al in a study carried out in SaSK patients and DM [17]. In another study which included 20 SaSK patients with DM, Silveiro et al concluded that the association of SaSK with DM leads to an increased prevalence of micro- and macroalbuminuria. Thus, SaSK diabetic patients presented a significantly higher rate of microalbuminuria (40\%) than SaSK non-diabetic patients $(17.6 \% ; \mathrm{P}=0.03)$ and type 2 DM patients (20\%; $\mathrm{P}=0.03)$. Also, SaSK diabetic patients had a higher proportion of macroalbuminuria (30\%) as compared with
SaSK non-diabetic patients $(6 \% ; \mathrm{P}=0.03)$ [18]. When analyzing the urinary albumin excretion rate and the SaSK duration, the development of albuminuria is faster in SaSK patients with DM versus SaSK patients with no DM (a median period of 5 years as compared to a median time of 30 years) [18]. Consistent with these data are the findings of a recent study performed by our team, in which we compared 84 SaSK patients with DM versus 84 patients only with DM. We observed that individuals with SK presented significantly higher proteinuria, but there were no differences regarding eGFR [19]. We concluded that there is a higher degree of renal injury in SaSK diabetic patients than in patients only with DM, even if this phenomenon is not reflected in the eGFR levels [19]. Furthermore, in this study we found significantly higher cardiovascular abnormalities in SaSK with DM versus patients only with DM (i.e. higher prevalence of arterial hypertension and coronary artery disease) [19].

\section{Congenital Solitary Kidney and Diabetes Mellitus}

To date, there are sparse data regarding the inter-relation between congenital SK (cSK) and DM. Most of the information currently available on the outcome of cSK highlights the fact that children born with a cSK have an increased risk of hypertension and CKD even from early adulthood [20]. By the age of 10 years, one in three cSK children has indicators of renal injury and more than $50 \%$ of the cSK children develop signs of renal injury by the age of 18 years, while $20-40 \%$ of patients with cSK require renal replacement therapy by the age of 30 years [20,21]. However, data about consequences due to superimposed DM to the cSK condition are only reported in few cohorts of cSK patients. Thus, Baturk et al. [22] found DM in $16.1 \%$ of the $31 \mathrm{cSK}$ patients who were evaluated. The presence of DM was significantly associated with CKD development in cSK patients $(\mathrm{P}=0.038)$ [22]. In contrast, in a recent Korean study which included 153 persons with cSK, the association of DM to the cSK status did not increase the risk of CKD development [23].

\section{Conclusion}

In SK individuals, renal function could be maintained within normal range through adaptive hyperfiltration and hypertrophy, but a superimposed "second hit" such as DM may lead to a poor kidney outcome. In LKD, de novo DM is associated with higher risk of ESRD in the late post donation period, a fact which implies a longer period of follow-up post donation and a rigorous selection of potential living donors. Solitary kidney patients should be always referred to a nephrologist in order to ensure an early diagnosis of renal damage and to decrease the risk of CKD progression.

\section{References}

1. Steffes MW, Brown DM, Mauer SM (1978) Diabetic glomerulopathy following unilateral nephrectomy in the rat. Diabetes 27(1): 35-41.

2. Whiteside C, Katz A, Cho C, Silverman M (1990) Diabetic glomerulopathy following unilateral nephrectomy in the dog. Clin Invest Med 13(5): 279286. 
3. Lopes GS, Lemos CC, Mandarim De Lacerda CA, Bregman R (2004) Effect of unilateral nephrectomy on renal function of diabetic rats. Histol Histopathol 19(4): 1085-1088.

4. Martins IJ (2017) Single gene inactivation with implications to diabetes and multiple organ dysfunction syndrome. J Clin Epigenet 3: 24

5. (2016) Global Observatory on Donation and Transplantation: Kidney Transplants.

6. Tantisattamo E, Dafoe DC, Reddy UG, Ichii H, Rhee CM, et al. (2019) Current management of patients with acquired solitary kidney. Kidney International Reports 4(9): 1205-1218.

7. Muzaale AD, Massie AB, Wang MC, Montgomery RA, McBride MA, et al. (2014) Risk of end-stage renal disease following live kidney donation. JAMA 311(6): 579-586.

8. Anjum S, Muzaale AD, Massie AB, Bae S, Luo X, et al. (2016) Patterns of end-stage renal disease caused by diabetes, hypertension, and glomerulonephritis in live kidney donors. Am J Transplant 16(12): 3540-3547.

9. Mjøen G, Hallan S, Hartmann A, Foss A, Midtvedt K, et al. (2014) Longterm risks for kidney donors. Kidney Int 86(1): 162-167.

10. Martins I (2017) Nutrition therapy regulates caffeine metabolism with relevance to NAFLD and induction of type 3 diabetes. Journal of Diabetes and Metabolic Disorders 4: 1-9.

11. Ibrahim HN, Berglund DM, Jackson S, Vock DM, Foley RN, et al. (2017) Renal consequences of diabetes after kidney donation. Am J Transplant 17(12): 3141-3148.

12. Ibrahim HN, Hostetter TH (1997) Diabetic nephropathy. JASN 8: $487-$ 493.

13. Lenihan CR, Busque S, Derby G, Blouch K, Myers BD, et al. (2015) Longitudinal study of living donor glomerular dynamics after nephrectomy. J Clin Invest 125(3): 1311-1318.
14. Zhang WJ, Wang ZY, Zhou WX, Yang NQ, Wang Y, et al. (2020) Identifying risk factors for chronic kidney disease stage 3 in adults with acquired solitary kidney from unilateral nephrectomy: a retrospective cohort study. BMC Nephrology 21(1): 397.

15. Malcolm JB, Bagrodia A, Derweesh IH, Mehrazin R, Diblasio CJ, et al. (2009) Comparison of rates and risk factors for developing chronic renal insufficiency, proteinuria and metabolic acidosis after radical or partial nephrectomy. BJU Int 104(4): 476-481.

16. Nielsen FS, Gall MA, Parving HH (1995) Acquired oligonephropathy and diabetic nephropathy. Am J Kidney Dis 26(6): 898-903.

17. Sampson MJ, Drury PL (1990) Development of nephropathy in diabetic patients with a single kidney. Diabet Med 7(3): 258-260.

18. Silveiro SP, da Costa LA, Beck MO, Gross JL (1998) Urinary albumin excretion rate and glomerular filtration rate in single-kidney type 2 diabetic patients. Diabetes Care 21(9): 1521-1524.

19. Gluhovschi C, Gluhovschi G, Gadalean F, Velciov S, Petrica L, et al. (2017) Renal function is similar in solitary kidneys from patients with and without diabetes. Nefrologia 37(2): 195-198.

20. Schreuder MF (2018) Life with one kidney. Pediatr Nephrol 33(4): 595604.

21. Sanna Cherchi S, Ravani P, Corbani V, Parodi S, Haupt R, et al. (2009) Renal outcome in patients with congenital anomalies of the kidney and urinary tract. Kidney Int 76(5): 528-533.

22. Basturk T, Koc Y, Ucar Z, Sakaci T, Ahbap E, et al. (2015) Renal damage frequency in patients with solitary kidney and factors that affect progression. Int J Nephrol 2015: 876907.

23. Kim S, Chang Y, Lee YR, Jung HS, Hyun YY, et al. (2019) Solitary kidney and risk of chronic kidney disease. Eur J Epidemiol 34(9): 879-888. 